\title{
Anisotropic susceptibility of the geometrically frustrated spin-chain compound $\mathrm{Ca}_{3} \mathrm{Co}_{2} \mathrm{O}_{6}$
}

\author{
Vincent Hardy, Delphine Flahaut, Raymond Frésard and \\ Antoine Maignan \\ Laboratoire CRISMAT, UMR CNRS-ENSICAEN(ISMRA) 6508, \\ 6 Bld. du Maréchal Juin, F-14050 Caen, France \\ E-mail: Vincent.Hardy@ensicaen.fr
}

\begin{abstract}
Ca}_{3} \mathrm{Co}_{2} \mathrm{O}_{6}$ is a system exhibiting a series of fascinating properties, including magnetization plateaus and remarkably slow dynamics at low- $T$. These properties are intimately related to the geometrical frustration, which results from a particular combination of features: (i) the chains are arranged on a triangular lattice; (ii) there is a large uniaxial anisotropy; (iii) the intrachain and interchain couplings are ferromagnetic and antiferromagnetic, respectively.

The uniaxial anisotropy is thus an issue of crucial importance for the analysis of the physical properties of $\mathrm{Ca}_{3} \mathrm{Co}_{2} \mathrm{O}_{6}$. However, it turns out that no precise investigation of this magnetic anisotropy has been performed so far. On the basis of susceptibility data directly recorded on single crystals, the present study reports on quantitative information about the anisotropy of $\mathrm{Ca}_{3} \mathrm{Co}_{2} \mathrm{O}_{6}$.

PACS numbers: 75.60.-d, 75.45.+j
\end{abstract}

Submitted to: J. Phys.: Condens. Matter 
Anisotropic susceptibility of the geometrically frustrated spin-chain compound $\mathrm{Ca}_{3} \mathrm{Co}_{2} \mathrm{O}_{6} 2$

\section{Introduction}

$\mathrm{Ca}_{3} \mathrm{Co}_{2} \mathrm{O}_{6}$ is a geometrically frustrated spin-chain compound which has attracted considerable attention in recent years, owing to a series of puzzling properties [1, 2, 3, 4, 5, 6, 7, 8, 9, 10, 11, 12]. The structure of this compound consists of chains made up of $\mathrm{CoO}_{6}$ trigonal prisms alternating with $\mathrm{CoO}_{6}$ octahedra, which run along the $c$-axis of the hexagonal cell [1]. These chains are separated by the Ca ions and they form an hexagonal lattice on the $a b$ plane (see inset of Fig. 1). The issue of the valence and spin states of the Co ions on these two sites has been the subject of intense controversies. Owing to a series of recent results [13, 14, 15, 16, 17], it is now widely accepted that (i) the Co ions are trivalent for both sites; (ii) owing to the difference in the crystalline electric field $(\mathrm{CEF})$, the $\mathrm{Co}^{3+}\left(3 d^{6}\right)$ ions are in the high-spin state for the prismatic sites $(S=2)$ whereas they are in the low-spin state $(S=0)$ for the octahedral sites. In other respects, it was early found that the intrachain coupling $J$ is ferromagnetic while the interchain coupling $J^{\prime}$ is antiferromagnetic [2]. Furthermore, $\mathrm{Ca}_{3} \mathrm{Co}_{2} \mathrm{O}_{6}$ was found to exhibit strongly anisotropic magnetic properties, the spins having a preferential orientation along the $c$ axis [4, 5]. Most probably, this last feature can be ascribed to the single-ion (uniaxial) anisotropy of the $S=2$ spins at the prismatic sites.

$\mathrm{Ca}_{3} \mathrm{Co}_{2} \mathrm{O}_{6}$ possesses a combination of features (i.e. triangular lattice with spins oriented along the chain direction and with an antiferromagnetic interchain coupling) which yields a situation of geometrical frustration [18. It is clear that this frustration plays a great role in the peculiar magnetic properties of $\mathrm{Ca}_{3} \mathrm{Co}_{2} \mathrm{O}_{6}$. For instance, the long-range ordering which takes place below $T_{N} \simeq 26 \mathrm{~K} \mathrm{[2]} \mathrm{was} \mathrm{found} \mathrm{to} \mathrm{be} \mathrm{closely}$ related to the "Partially Disordered Antiferromagnetic" state (PDA) [3] proposed by

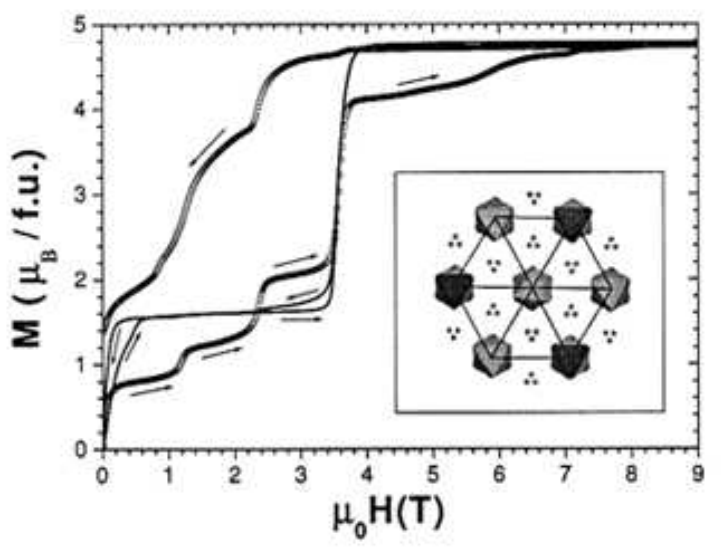

Figure 1. Hysteresis loops $(H \| c)$ recorded with a sweep rate of $0.1 \mathrm{~T} / \mathrm{min}$. at $2 \mathrm{~K}$ (circles) and $10 \mathrm{~K}$ (solid line). The arrows indicate the direction of the field variation. The inset shows a projection of the structure along the hexagonal $c$-axis (the dark and light polyhedra represent $\mathrm{CoO}_{6}$ trigonal prisms and $\mathrm{CoO}_{6}$ octahedra, respectively; the grey circles represent the $\mathrm{Ca}$ ions; the solid lines emphasize the triangular arrangement of the chains in the $a b$ plane). 
Mekata [19] about the $\mathrm{ABX}_{3}$ compounds, another family of geometrically frustrated spin-chains [18]. In the PDA state, two over three chains are antiferromagnetically coupled (with long-range order along each of them), while the third one is left incoherent (i.e. without long-range order along its direction and no correlation with its neighbors). Among the peculiar magnetic properties of $\mathrm{Ca}_{3} \mathrm{Co}_{2} \mathrm{O}_{6}$, one can mention (i) the appearance of a spin freezing at $T_{S F}\left(=T_{f=0}\right) \sim 8 \mathrm{~K}$ [3, 6, 17, leading to a pronounced frequency dependence of the susceptibility $\left(\Delta T_{f} / T_{f}\right) / \Delta \log f \simeq 0.17$ ) [5, 12]; (ii) for $T<T_{S F}$, the existence of magnetization steps with a roughly constant field spacing [4, 5, 20] and the appearance of a saturation in the spin-relaxation time, these features having led us to suggest the possibility of a phenomenon of Quantum Tunneling of the Magnetization (QTM) [20, 12].

It can be emphasized that the geometrical frustration in $\mathrm{Ca}_{3} \mathrm{Co}_{2} \mathrm{O}_{6}$ is strongly related to the existence of a pronounced uniaxial anisotropy. In spite of this, we observe that no precise quantitative analysis of this magnetic anisotropy has been performed so far. It is the goal of the present study to extract quantitative information about the anisotropy of $\mathrm{Ca}_{3} \mathrm{Co}_{2} \mathrm{O}_{6}$.

\section{Experimental details}

Single crystals of $\mathrm{Ca}_{3} \mathrm{Co}_{2} \mathrm{O}_{6}$ were grown according to the following method: a mixture of $\mathrm{Ca}_{3} \mathrm{Co}_{4} \mathrm{O}_{9}$ and $\mathrm{K}_{2} \mathrm{CO}_{3}$, in a weight ratio of $1 / 7$, was heated at $950{ }^{\circ} \mathrm{C}$ for 50 hours in an alumina crucible in air; then, the cooling was performed in two steps, first down to $930{ }^{\circ} \mathrm{C}$ at $10{ }^{\circ} \mathrm{C} / \mathrm{h}$ and then down to room temperature at $100{ }^{\circ} \mathrm{C} / \mathrm{h}$. This procedure leads to crystals having a needle-like shape (with the $c$ axis along the longest dimension) which is convenient for orientation purposes. These crystals can be quite long (typically $4 \mathrm{~mm}$ ) but they are thin (less than $0.5 \mathrm{~mm}$ ). Accordingly, we performed the measurements on an assembly of crystals. Owing to their shape, we emphasize that the alignment between them, as well as with respect to the field direction (i.e. $c$ axis either parallel or perpendicular to the field direction) can be precisely achieved. Moreover, the measurements for both orientations were carried out with the same mounting system - just by rotating the set of crystals inside the sample rod - in order to keep constant the residual background signal.

Curves of magnetization as a function of $T$ were recorded in $0.1 \mathrm{~T}$, for both orientations, by using a Superconducting Quantum Interference Device magnetometer (MPMS, Quantum Design). We checked that, with $0.1 \mathrm{~T}$, we are in the linear regime of the $M(H)$ curves for both orientations and over the whole investigated temperature range, $50<T<300 \mathrm{~K}$ (i.e. $T>>T_{N}$ ). In what follows, one considers the susceptibility curves obtained by dividing the magnetization by the measuring field, leading to $\chi_{\|}(T)$ (i.e. with $H \| c$ ) and $\chi_{\perp}(T)$ (i.e. with $H \perp c$ ). Two observations support the reliability of these data: first, we found that the $\chi_{\|}(T)$ is in perfect agreement with that previously obtained using bigger crystals (of different shape); second, the powder-like data obtained by calculating $\chi_{p}=\left(\chi_{\|}+2 \chi_{\perp}\right) / 3$ is found to be superimposed on the susceptibility curve 
Anisotropic susceptibility of the geometrically frustrated spin-chain compound $\mathrm{Ca}_{3} \mathrm{Co}_{2} \mathrm{O}_{6} 4$ registered on a ceramic (see Fig. 2).

\section{Results and Discussions}

Figure 2 shows the $\chi_{\|}(T)$ and $\chi_{\perp}(T)$ data - as well as the derived $\chi_{p}(T)$ curve - along with data recorded on a ceramic sample and on bigger crystals with $H \| c$. These data well exhibit the considerable magnetic anisotropy of this compound. To be more quantitative, however, one needs a proper modelization of the susceptibilities. It turns out that it is far from being obvious in the case of $\mathrm{Ca}_{3} \mathrm{Co}_{2} \mathrm{O}_{6}$ which gathers several particularities.

As previously discussed, we can assume that the anisotropy of $\mathrm{Ca}_{3} \mathrm{Co}_{2} \mathrm{O}_{6}$ is basically a single-ion feature, originating from a combination of the CEF and spin-orbit effects. We can also simply consider a Heisenberg form for the intrachain coupling, while the interchain coupling (much weaker) can be neglected in a first approximation. In other respects, we note that a spin chain compound would require in principle a specific treatment of the magnetic coupling in order to account for the enhanced fluctuation effects in 1D systems.

To the best of our knowledge, there is no model in the literature which takes into account all the features of $\mathrm{Ca}_{3} \mathrm{Co}_{2} \mathrm{O}_{6}$. In what follows, we will consider two modelizations which can be applicable - to some extent- to the case of $\mathrm{Ca}_{3} \mathrm{Co}_{2} \mathrm{O}_{6}$. We emphasize that each of these models only accounts for a part of the features of $\mathrm{Ca}_{3} \mathrm{Co}_{2} \mathrm{O}_{6}$. It also deserves to be noted that they correspond to different approaches that can be complementary in the analysis of magnetism.

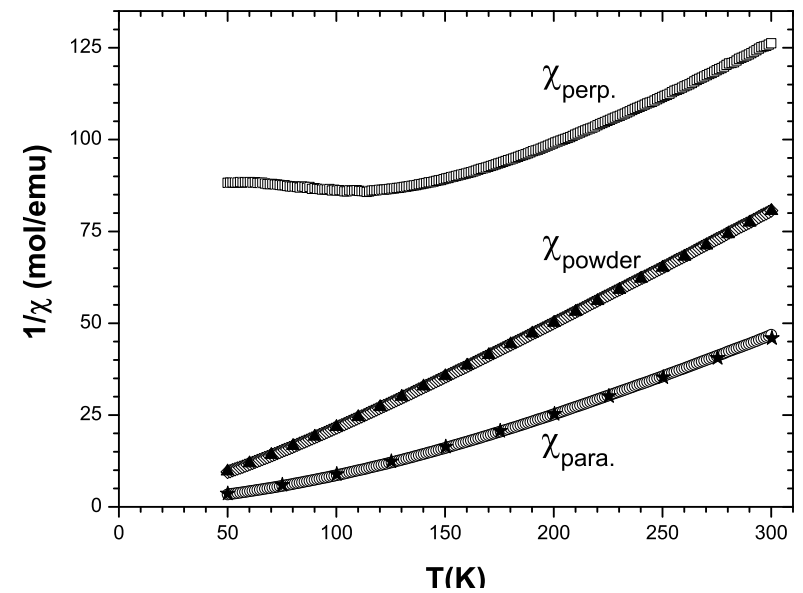

Figure 2. Susceptibility data recorded on single crystals of $\mathrm{Ca}_{3} \mathrm{Co}_{2} \mathrm{O}_{6}$, with the magnetic field applied either along the $c$-axis ( $\chi_{\text {para }}$ referred to as $\chi_{\|}$in the text) or perpendicular to the c-axis ( $\chi_{\text {perp }}$ referred to as $\chi_{\perp}$ in the text). Also shown is the corresponding "powder" data derived from the general expression $\chi_{p}=\left(\chi_{\|}+2 \chi_{\perp}\right) / 3$. The small filled triangles represent the data directly recorded on a ceramic sample. The small filled stars represent the data previously recorded on another set of crystals (different morphology with a larger size) with $H \| c$. 
Anisotropic susceptibility of the geometrically frustrated spin-chain compound $\mathrm{Ca}_{3} \mathrm{Co}_{2} \mathrm{O}_{6} 5$

\subsection{First model}

We found in the literature a model which made a precise treatment of the on-site magnetism in a situation very close to ours. This work carried out by Parkin and Friend [21] dealt with iron intercalates of dichalcogenides, with $\mathrm{Fe}^{2+}$ (i.e., $3 \mathrm{~d}^{6}$ like $\mathrm{Co}^{3+}$ ) in a trigonally distorted anionic environment. It turns out that the cubic part of the CEF splits the ${ }^{5} D$ ground state, and generates a $\Gamma_{5}$ triplet at lower energy, which can be represented by a fictitious orbital angular momentum $\widetilde{\mathbf{L}}$. On this basis, Parkin and Friend [21] performed a rigorous analysis by considering the following single-site Hamiltonian:

$$
H_{i}=-k_{B} \delta \widetilde{L}_{z, i}^{2}-k_{B} \lambda \widetilde{\mathbf{L}}_{i} \cdot \mathbf{S}_{i}+\mu_{B}\left(-\widetilde{\mathbf{L}}_{i}+2 \mathbf{S}_{i}\right) \mathbf{B} .
$$

The first term corresponds to the effect of the non-cubic part of the CEF, the second term is the spin-orbit (SO) coupling, and the last term is the basic form of the Zeeman energy. For our compound with such an Hamiltonian, $\delta$ is positive while $\lambda$ is negative. There is no magnetic coupling in this model but we note that a spin-spin interaction can be added - if necessary - by using a mean-field (MF) approximation (even though we know such a MF treatment is poorly suited to 1D systems).

Using the eigenfunctions and associated energies, Parkin and Friend calculated the susceptibilities using the Van Vleck formula. Extending their approach, we took into account all the levels of the lower triplet (whereas only the two lowest are considered in Ref. [21]), but it actually makes almost no difference. The expressions found for the susceptibilities can be written as:

$$
\begin{aligned}
& \chi_{\|}=\frac{N \mu_{B}^{2} S(S+1)}{3 k_{B}} \frac{1}{T}\left[\frac{f_{\|}}{Z}\right] \\
& \chi_{\perp}=\frac{N \mu_{B}^{2} S(S+1)}{3 k_{B}} \frac{1}{T}\left[\frac{f_{\perp}}{Z}\right]
\end{aligned}
$$

where

$$
\begin{aligned}
f_{\|}= & 25 \exp (-2 s)+9 \exp (-s)+1+\exp (s)+ \\
& 9 \exp (2 s)+16 \exp \left(\frac{2 s^{2}}{p}+p\right)+4 \exp \left(\frac{5 s^{2}}{p}+p\right), \\
f_{\perp}= & (-8 / s) \exp (-2 s)-(4 / s) \exp (-s)+(4 / s) \exp (s)+ \\
& (8 / s) \exp (2 s)+16 \exp \left(\frac{2 s^{2}}{p}+p\right)+4 \exp \left(\frac{5 s^{2}}{p}+p\right), \\
Z= & 2[\exp (-2 s)+\exp (-s)+1+\exp (s)+\exp (2 s)+ \\
& \left.\exp \left(\frac{2 s^{2}}{p}+p\right)+\exp \left(\frac{5 s^{2}}{p}+p\right)+1 / 2 \exp \left(\frac{6 s^{2}}{p}+p\right)\right],
\end{aligned}
$$


Anisotropic susceptibility of the geometrically frustrated spin-chain compound $\mathrm{Ca}_{3} \mathrm{Co}_{2} \mathrm{O}_{6} 6$

with $s=\lambda / T$ and $p=-\delta / T$. All others parameters have their usual meaning.

It can be emphasized that the exact value of $\delta$ has only a tiny influence on the final results (we used $\delta=-100 \mathrm{~K}$ hereafter). Roughly speaking, one can consider that the main role of the non cubic part of the CEF is to split the $\Gamma_{5}$ triplet, which allows the SO to generate anisotropy. In other respects, it must be realized that $\lambda$ cannot really be regarded as a free parameter in these expressions, since the spin orbit parameter is supposed to be an intrinsic characteristic of each ion, not expected to vary significantly with the environment. In the case of $\mathrm{Co}^{3+}, \lambda$ must be close to $-145 \mathrm{~cm}^{-1}$ [22. Accordingly, it deserves to be noted that there is almost no adjustable parameter in this model, apart from a possible correction related to $J$.

Figure 3 shows the raw data, along with the curves calculated for two values of $\lambda$. With the expected value $\lambda=-145 \mathrm{~cm}^{-1}$, the calculated curves do not lie far from the experimental ones, they have the right shape in the high- $T$ range, but there is a sizeable shift from the data. Moreover, the model cannot account for the existence of a minimum in $1 / \chi_{\perp}(T)$, as it is visible in the data around $120 \mathrm{~K}$. We also observed that adding a correction related to the intrachain coupling can yield a very nice fitting for $\chi_{\|}(T)$ but not for $\chi_{\perp}(T)$. Strikingly, it can be noticed that remarkable fittings of both curves can be obtained with $\lambda=-165 \mathrm{~cm}^{-1}$. As previously discussed, however, such a significant departure from the free ion value of $\lambda$ is suspect. In conclusion, one must state that this model cannot well describe the magnetic anisotropy of $\mathrm{Ca}_{3} \mathrm{Co}_{2} \mathrm{O}_{6}$, even though it well illustrates the leading role of the spin orbit coupling.

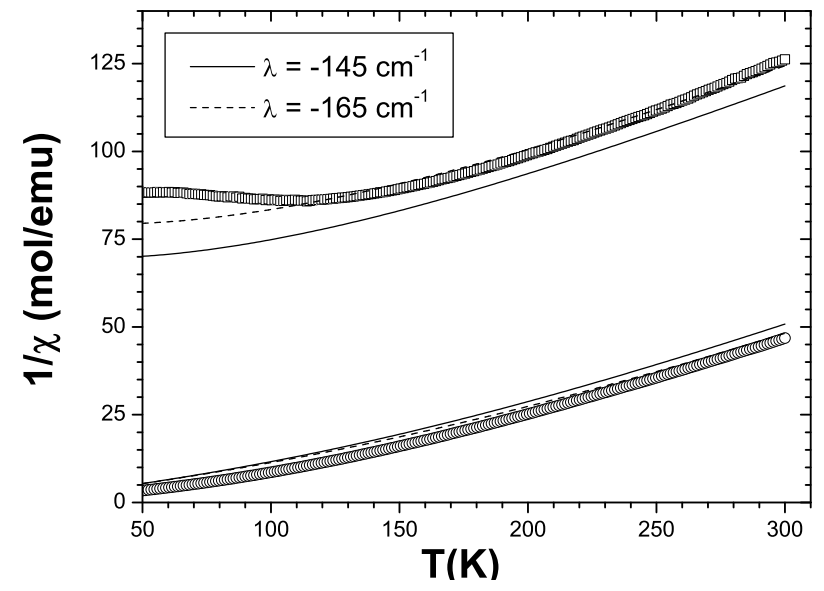

Figure 3. Experimental $\chi_{\|}(T)$ and $\chi_{\perp}(T)$ curves, together with the calculations using the model of Parkin and Friend with $\lambda=-145 \mathrm{~cm}^{-1}$ (solid lines) and $\lambda=-165$ $\mathrm{cm}^{-1}$ (dashed lines). 
Anisotropic susceptibility of the geometrically frustrated spin-chain compound $\mathrm{Ca}_{3} \mathrm{Co}_{2} \mathrm{O}_{6} 7$

\subsection{Second model}

Let us now consider a very different approach, approximating the susceptibility in the form of series expansion. This general method discards the specificities of the system under investigation (in contrast to the previous model), but it was found to be powerful in many cases, even though it is by definition limited to the high- $T$ part of the data (i.e. applicable only for $k T$ larger than all the energy terms relevant to the system). Yosida [23] has derived such a series expansion which appears to be suitable to the case of $\mathrm{Ca}_{3} \mathrm{Co}_{2} \mathrm{O}_{6}$, in the sense that it accounts for both a magnetic coupling $\mathrm{J}$ and an anisotropy parameter D. The starting point is an Hamiltonian of the form:

$$
H_{i}=-k_{B} D S_{z, i}^{2}-2 k_{B} J \sum_{j \neq i} \mathbf{S}_{i} \mathbf{S}_{j}+g \mu_{B} \mathbf{S}_{i} \mathbf{B},
$$

where the index $j$ refers to the first neighboring spins. In such a picture, the Zeeman term involves a Landé factor (which can be an adjustable parameter), while the uniaxial anisotropy appears as an effective term applied to the spin. Even though it can carry some ambiguities, this form of Hamiltonian is the most common to deal with uniaxial anisotropy, and it is very useful in practice. We underline that the $\delta$ parameter of the previous model strongly differs from $D$ in the above equation (this latter parameter incorporating both the CEF and SO effects).

The formula derived by Yosida can be written as follows:

$$
\begin{aligned}
& \chi_{\|}=\frac{C}{T-\theta}\left[1+2 \frac{Q}{T}\right], \\
& \chi_{\perp}=\frac{C}{T-\theta}\left[1-\frac{Q}{T}\right],
\end{aligned}
$$

In these expressions,

- $C$ is the usual Curie constant

$$
C=\frac{N g^{2} \mu_{B}^{2} S(S+1)}{3 k_{B}}
$$

- $\theta$ is the characteristic temperature associated to $J$ (i.e. the Curie-Weiss temperature)

$$
\theta=\frac{2 z J S(S+1)}{3}
$$

(here $z$ is the number of nearest neighbors, i.e. 2 in the case of intrachain coupling)

- $Q$ is a characteristic temperature associated to $D$

$$
Q=D\left[\frac{2 S(S+1)}{15}-\frac{1}{10}\right]
$$

Note that, in our case, $\theta>0$ and $Q>0$.

In Eqs (8-9), they are three free parameters which should be adjusted to best fit to both the $\chi_{\|}(T)$ and $\chi_{\perp}(T)$ data. Actually, one can also use combinations of $\chi_{\|}(T)$ 


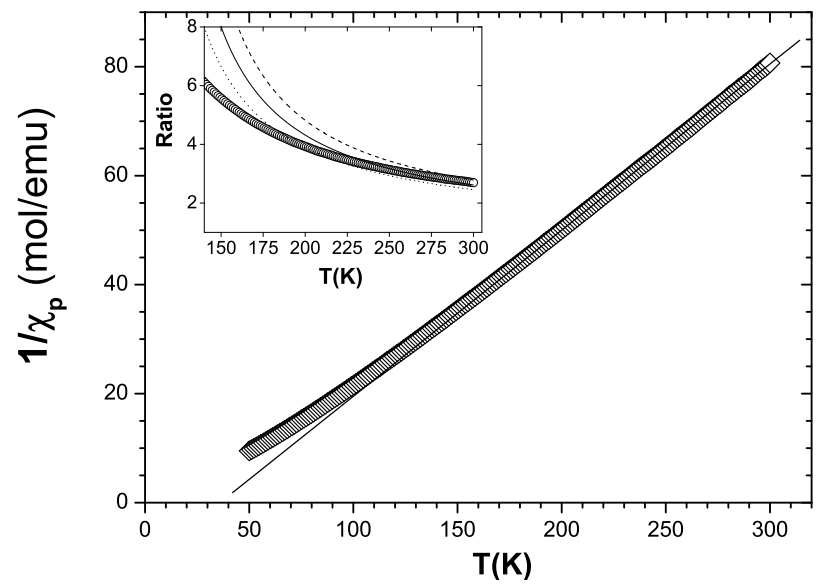

Figure 4. The main panel shows the $1 / \chi_{p}(T)$ curve derived from the data (see text), which exhibits a linear behavior at high temperature (solid line). The inset shows the temperature dependence of the susceptibility ratio $\left(\chi_{\|} / \chi_{\perp}\right)$, together with the expectations of the Yosida's model for several values of $D$ : from bottom to top, $D=140 \mathrm{~K}$ (dotted line), $D=150 \mathrm{~K}$ (solid line) and $D=160 \mathrm{~K}$ (dashed line).

and $\chi_{\perp}(T)$ allowing to isolate the influence of some of these parameters. For instance, it can be noted that the parameter $D$ disappears in the powder formula, yielding the usual form

$$
\chi_{p}=\frac{\chi_{\|}+2 \chi_{\perp}}{3}=\frac{C}{T-\theta} .
$$

As shown in Fig. 4, such a behavior is found to be well obeyed by the data. Fitting to the data in the range $200-300 \mathrm{~K}$ leads to $g \simeq 2.08$ and $J \simeq 4.5 \mathrm{~K}$.

Furthermore, the ratio $\left(\chi_{\|} / \chi_{\perp}\right)$ is expected to only depend on $D$

$$
\frac{\chi_{\|}}{\chi_{\perp}}=\frac{1+2 \frac{Q}{T}}{1-\frac{Q}{T}}
$$

Inset of Fig. 4 shows that there is a restricted range of $D$ values - around $150 \mathrm{~K}$ which leads to a reasonable fitting in the high- $T$ range.

The $\chi_{\|}(T)$ and $\chi_{\perp}(T)$ curves, corresponding to $g=2.08, D=150 \mathrm{~K}$ and $J=4.5 \mathrm{~K}$, are reported in Fig. 5. The agreement with the data is far from being perfect, but it is reasonable if one considers that the same set of parameters must fit to two curves (not to mention that perfect fittings are obtained if one authorizes different sets of parameters for each curve). Probably, a part of the deviation from the data can be attributed to the fact that the temperatures of the fitting range are not much larger that $D$. Moreover, this model provides a rough description of the magnetic coupling since the 1D character is only taken into account through the value of $z(=2)$. 


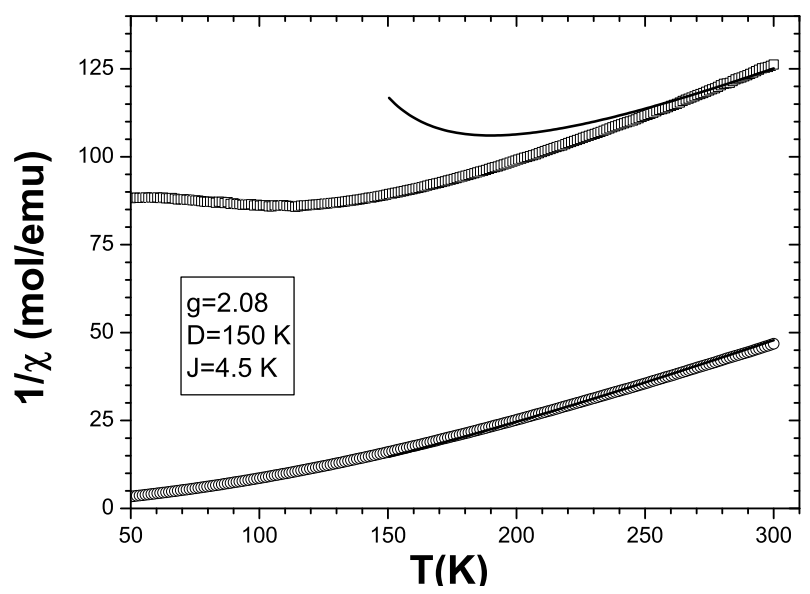

Figure 5. Experimental $\chi_{\|}(T)$ and $\chi_{\perp}(T)$ curves, together with the calculations using the Yosida's model for the displayed values of parameters (according to the assumptions of this model, the calculations are limited to the high- $T$ range).

\section{Conclusion}

The anisotropic susceptibility of the geometrically frustrated spin chain $\mathrm{Ca}_{3} \mathrm{Co}_{2} \mathrm{O}_{6}$ has been measured on single crystals. Two models of the literature have been used to analyze these data. None of them was found to be perfectly satisfying. However, the first one clearly pointed to the role of the SO in this anisotropy [21], while the second one provided us with a reliable estimate of the effective anisotropy parameter $D \simeq 150 \mathrm{~K}$ [23. This value is of importance to go further into the analysis of the peculiar magnetic properties exhibited by this compound.

The present study also pointed to the issues that should be addressed in priority to better describe $\chi_{\|}(T)$ and $\chi_{\perp}(T)$ in $\mathrm{Ca}_{3} \mathrm{Co}_{2} \mathrm{O}_{6}$ : (i) owing to the large values of $D$, it is necessary to develop a reliable model for $T<D$; (ii) since the intrachain coupling seems to have an impact even at large $T$ (when considering a MF approach), it would be valuable to consider a modelization accounting for the $1 \mathrm{D}$ character of this compound. These developments are presently under way.

\section{References}

[1] Fjellvåg H, Gulbrandsen E, Aasland S, Olsen A and Hauback B 1996 J. Solid State Chem. 124 190

[2] Aasland S, Fjellvåg H and Hauback B 1997 Solid State Comm. 101187

[3] Kageyama H, Yoshimura K, Kosuge K, Mitamura H and Goto T 1997 J. Phys. Soc. Jpn. 661607

[4] Kageyama H, Yoshimura K, Kosuge K, Azuma M, Takano M, Mitamura H and Goto T $1997 \mathrm{~J}$. Phys. Soc. Jpn. 663996

[5] Maignan A, Michel C, Masset A C, Martin C and Raveau B 2000 Eur. Phys. J. B 15657

[6] Hardy V, Lambert S, Lees M R and McK Paul D Phys. Rev. B 68014424 
Anisotropic susceptibility of the geometrically frustrated spin-chain compound $\mathrm{Ca}_{3} \mathrm{Co}_{2} \mathrm{O}_{6} 10$

[7] Hardy V, Lees M R, Maignan A, Hébert S, Flahaut D, Martin C and McK Paul D 2003 J. Phys.: Condens. Matter 155737

[8] Rayaprol S, Sengupta K and Sampathkumaran E V 2003 Solid State Comm. 12879

[9] Frésard R, Laschinger C, Kopp T and Eyert V 2004 Phys. Rev. B 69 140405(R)

[10] Hardy V, Lees M R, Petrenko O A, McK Paul D Flahaut D, Hébert S and Maignan A 2004 Phys. Rev. B 70064424

[11] Flahaut D, Maignan A, Hébert S, Martin C, Retoux R and Hardy V 2004 Phys. Rev. B 70094418

[12] Hardy V, Flahaut D, Lees M R and Petrenko O A 2004 Phys. Rev. B 70214439

[13] Whangbo M H, Dai D, Koo H J and Jobic S 2003 Solid State Comm. 125413

[14] Sampathkumaran E V, Fujiwara N, Rayaprol S, Madhu P K and Uwatoko Y 2004 Phys. Rev. B 70014437

[15] Eyert V, Laschinger C, Kopp T and Frésard R 2004 Chem. Phys. Lett. 385249

[16] Takubo K, Mizokawa T, Hirata S, Son J Y, Fujimori A, Topwal D, Sarma D D, Rayapol S and Sampathkumaran E V 2005 Phys. Rev. B 71073406

[17] Wu H, Haverkort M W, Hu Z, Khomskii D I and Tjeng L H 2005 Phys. Rev. Lett. 95186401

[18] Collins M F and Petrenko O A 1997 Can. J. Phys. 75605

[19] Mekata M 1977 J. Phys. Soc. Jpn. 4276 Mekata M and Adachi K 1978 J. Phys. Soc. Jpn. 44806

[20] Maignan A, Hardy V, Hébert S, Drillon M, Lees M R, Petrenko O A, McK Paul D and Khomskii D 2004 J. Mater. Chem. 141231

[21] Parkin S S P and Friend R H 1980 Philosophical Magazine B 4165

[22] Abragam A and Bleaney B 1970 Electron Paramagnetic Resonance of Transition Ions (Oxford University Press, London).

[23] Yosida K 1951 Progress of Theoretical Physics 5, 691 (1951). 